\title{
Magnetic Properties and Microstructure of Nanocrystalline NdFeB Magnets Fabricated by a Modified Hot Working Process
}

\author{
Hyoung-Tae Kim ${ }^{1,4}$, Yoon-Bae Kim ${ }^{1, *}$, G. A. Kapustin'², Woo-Yong Jeon ${ }^{3}$ and Hak-Shin Kim \\ ${ }^{\prime}$ Korea Research Institute of Standards and Science, Daejeon 305-600, Korea \\ ${ }^{2} R R C$, Kurchatov Institute, Moscow, Russia \\ ${ }^{3}$ Kwangyang Colledge, Kwangyang 545-703, Korea \\ ${ }^{4}$ Research Center of Advanced Material Development, Chonbuk Natl Univ., Jeonju 561-756, Korea
}

(Received 9 December 2002)

\begin{abstract}
Magnetic properties, microstructure and texture of $\mathrm{NdFeB}$ magnets fabricated by a modified hot working process from commercial melt-spun powders (Magnequench; MQPA, MQPB and MQPB+) have been investigated. The hot-pressed isotropic magnet made from MQPA powder, which contains higher Nd content than that of MQPB or MQPB+, shows higher coercivity. The magnet also shows homogenous and fine grains with higher coercivity for higher consolidation pressure. The hot-deformed MQPA magnet shows a strong anisotropy along the press direction with homogeneous platelet $\mathrm{Nd}_{2} \mathrm{Fe}_{14} \mathrm{~B}$ grains of $50 \sim 100 \mathrm{~nm}$ in thickness and 200 500 nm in length. The hot-deformed MQPB+ magnet, however, shows low remanence and low coercivity. The microstructure of the magnet consists of two areas; undeformed $\mathrm{Nd}_{2} \mathrm{Fe}_{14} \mathrm{~B}$ grains and well-aligned but large grains with $3 \sim 4 \mu \mathrm{m}$ in length. Low Nd content attributes to the formation of the two different area.
\end{abstract}

Key words : CAPA process, $\mathrm{NdFeB}$ magnet, Hot press, Hot deformation, permanent magnet

\section{Introduction}

It is well known that the magnetic alignment in a meltspun consolidated NdFeB alloy is induced by hot working process, i.e. hot-press and die-upset [1-3]. Plastic deformation of hot-pressed precursors caused by die-upset allows equiaxed grains to platelets and improves the magnetic properties due to the development of the c-axis texture along the press direction

For hot-working, it is necessary to heat melt-spun powders or isotropic compacts to a temperature near the melting point of Nd-rich phase. However, heating the materials for a long time leads to the decrease of productivity and deterioration of hard magnetic properties. Thus, the alternative consolidation techniques, which enable the production of $\mathrm{NdFeB}$ magnets while avoiding prolonged heating, have been investigated. Shock compression [4-6] and spark plasma sintering [7-9] have the advantage such as high sintering speed and high reproducibility.

Recently, we have studied a modified hot-working

*Corresponding author: Tel: +82-42-868-5161, e-mail: ybkim@ kriss.re.kr method to fabricate textured NdFeB magnets. The method utilizes direct joule heating during the consolidation of powder and plastic deformation of hot-pressed precursors $[10,11]$. In this work, we compare the magnetic properties, microstructures and texture of $\mathrm{NdFeB}$ magnets obtained by the process for the commercial NdFeB powders which contain different $\mathrm{Nd}$ content.

\section{Experimental Method}

Starting materials used in this experiment were commercial melt-spun NdFeB powders, MQPA, MQPB and MQPB+ (Magnequench Co.). Table 1 shows the nominal composition of the powders. The MQPA powder showed an irregular flaky appearance with the average particle

Table 1. The nominal composition (wt.\%) of the melt-spun commercial NdFeB powders. The parenthesized passage is atomic percentage.

\begin{tabular}{lccccc}
\hline \hline Powder & $\mathrm{Fe}$ & $\mathrm{Nd}$ & $\mathrm{B}$ & $\mathrm{Co}$ & Dy \\
\hline MQPA & $68.6(80.9)$ & $30.5(13.8)$ & $0.9(5.3)$ & & \\
MQPB & $66.6(77.3)$ & $27(12.2)$ & $0.9(5.2)$ & $5(5.2)$ & $0.5(0.2)$ \\
MQPB+ & $66.6(77.3)$ & $27(12.2)$ & $0.9(5.2)$ & $5(5.2)$ & $0.5(0.2)$ \\
\hline
\end{tabular}




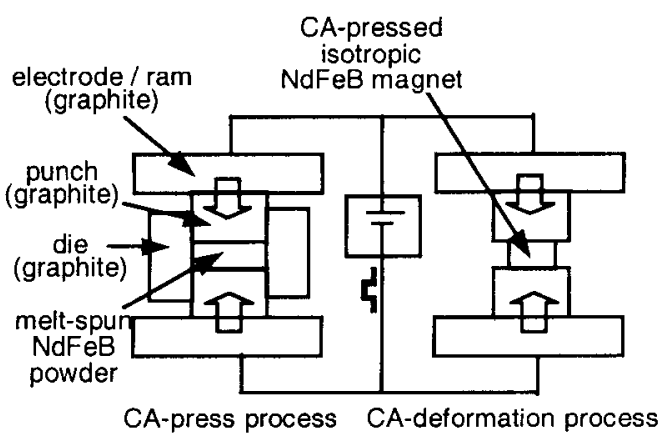

(a) CAPA processes

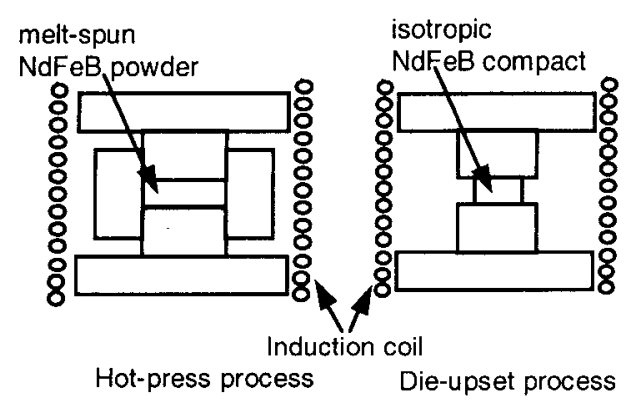

(b) Conventional hot-working processes

Fig. 1. The schematic diagram of the conventional hot working process and the CAPA process.

size of $230 \mu \mathrm{m}$. For MQPB and MQPB+, the average particle size were 260 and $240 \mu \mathrm{m}$, respectively.

As compared with conventional hot-working process in Fig. 1, about $20 \mathrm{~g}$ of powders were poured into a graphite die of the modified hot-working machine, and the system was evacuated to $3 \times 10^{-3}$ Torr. For consolidation, as shown in Fig. 1, the hydraulic pressure $\left(P_{\mathrm{a}}\right)$ and direct current $\left(I_{\mathrm{dc}}\right)$ were applied simultaneously between the upper and lower graphite punches under Ar atmosphere. The consolidation process was monitored by a LVDT which detects the shrinkage of the specimen. For the convenience, this process is referred as CA-press (currentapplied press), and the fully dense isotropic $\mathrm{NdFeB}$ magnets (20-mm-diameter with 7-mm-length) were obtained. In order to endow magnetic anisotropy, a $1 / 4$ piece of the CA-pressed magnet was placed between the punches in an open die and pressed to allow plastic deformation while current is flowing. This procedure is referred in this experiment as CA-deformation (current applied deformation) process.

After magnetizing the specimens in a pulsed field of $\sim 7600 \mathrm{kA} / \mathrm{m}(95 \mathrm{kOe})$, the magnetic properties were measured by a hysteresisgraph system with a maximum applied field of $\sim 1600 \mathrm{kA} / \mathrm{m}$ ( $20 \mathrm{kOe})$. The phase of specimen was examined by an $\mathrm{x}$-ray diffractometer with $\mathrm{Cu} K_{\alpha}$ radiation. Pole figures corresponding to (006) reflections were taken using a goniometer at a fixed angle
$2 \theta=44.5^{\circ}$. The tilting angle $(\alpha)$ and rotation angle $(\beta)$ varied from $0^{\circ}$ to $80^{\circ}$ in steps $5^{\circ}$ and from $0^{\circ}$ to $360^{\circ}$ in steps $20^{\circ}$, respectively. The microstructure of specimen was observed by scanning electron microscopy (SEM) and field emission scanning electron microscopy (FESEM, in KBSI).

\section{Results and Discussion}

Fig. 2 shows the demagnetization curves and SEM images of the CA-pressed magnets obtained from MQPA powder by applying $I_{\mathrm{dc}}=2500 \mathrm{~A}$ and $P_{\mathrm{a}}=10 \sim 70 \mathrm{MPa}$. The $B_{\mathrm{r}}$ of all specimens is higher than would be expected for a complete isotropic material $(\sim 0.8 \mathrm{~T})$ indicating the partial alignment of the c-axis of $\mathrm{Nd}_{2} \mathrm{Fe}_{14} \mathrm{~B}$ along the press direction. The SEM image for the cross sectional surface of the specimens shows that the application of low pressure, $P_{\mathrm{a}}=10 \mathrm{Mpa}$, results in an inhomogeneous microstructure with abnormal grain growth to several microns rectangular (Fig. 2(a)). With increasing the pressure to 30 $\mathrm{MPa}$, a homogeneous and fine grain structure is obtained, and the coercivity increases to $1103 \mathrm{kA} / \mathrm{m}(\sim 13.9 \mathrm{kOe})$. Further increase of $P_{\mathrm{a}}$ higher than $50 \mathrm{MPa}$, almost the same or higher ${ }_{\mathrm{i}} H_{\mathrm{c}}$ than that of raw powder is obtained with the uniform distribution of sub-micron scaled grains (See Fig. 2(c) and (d)).

Fig. 3 shows the demagnetization curves of the isotropic and anisotropic NdFeB magnets obtained by the CA-press $\left(I_{\mathrm{dc}}=2500 \mathrm{~A}, P_{\mathrm{a}}=70 \mathrm{MPa}\right)$ and the subsequent CAdeformation $\left(I_{\mathrm{dc}}=2000 \mathrm{~A}, P_{\mathrm{a}}=50 \mathrm{MPa}\right)$, respectively. The ${ }_{\mathrm{i}} H_{\mathrm{c}}$ of CA-pressed MQPA magnet is $1416 \mathrm{kA} / \mathrm{m}$ $(\sim 17.8 \mathrm{kOe})$ which is higher than the coercivity of raw powder. However, the ${ }_{\mathrm{i}} H_{\mathrm{c}}$ of the CA-pressed MQPB and MQPB+ magnets are only about $40 \sim 60 \%$ of their raw powders (See Table 2). Microstructural observation on the specimens showed no serious difference in their grain size $(100 \sim 400 \mathrm{~nm})$, and the low coercivity of the CA-pressed MQPB and MQPB+ magnets are thought due to the low amount of non-magnetic phase (Nd-rich phase) because of their low Nd content in raw powders.

After CA-deformation, the specimens show strong anisotropic property with enhanced remanence along the press direction. However, the $B_{\mathrm{r}}$ of CA-deformed MQPB and $\mathrm{MQPB}+$ are considerably lower than that of CA-deformed MQPA. The XRD patterns for the specimens, as shown in Fig. 4, are also with strong (006) and (105) reflections, and the coincidence with magnetic anisotropy are unclear within its resolution. X-ray pole figure gives the more accurate information on texture. Fig. 5 shows the (006) pole figures for the $\mathrm{CA}$-deformed anisotropic specimens. Here, the inner most and the $2^{\text {nd }}$ concentric circle is for $\alpha$ 


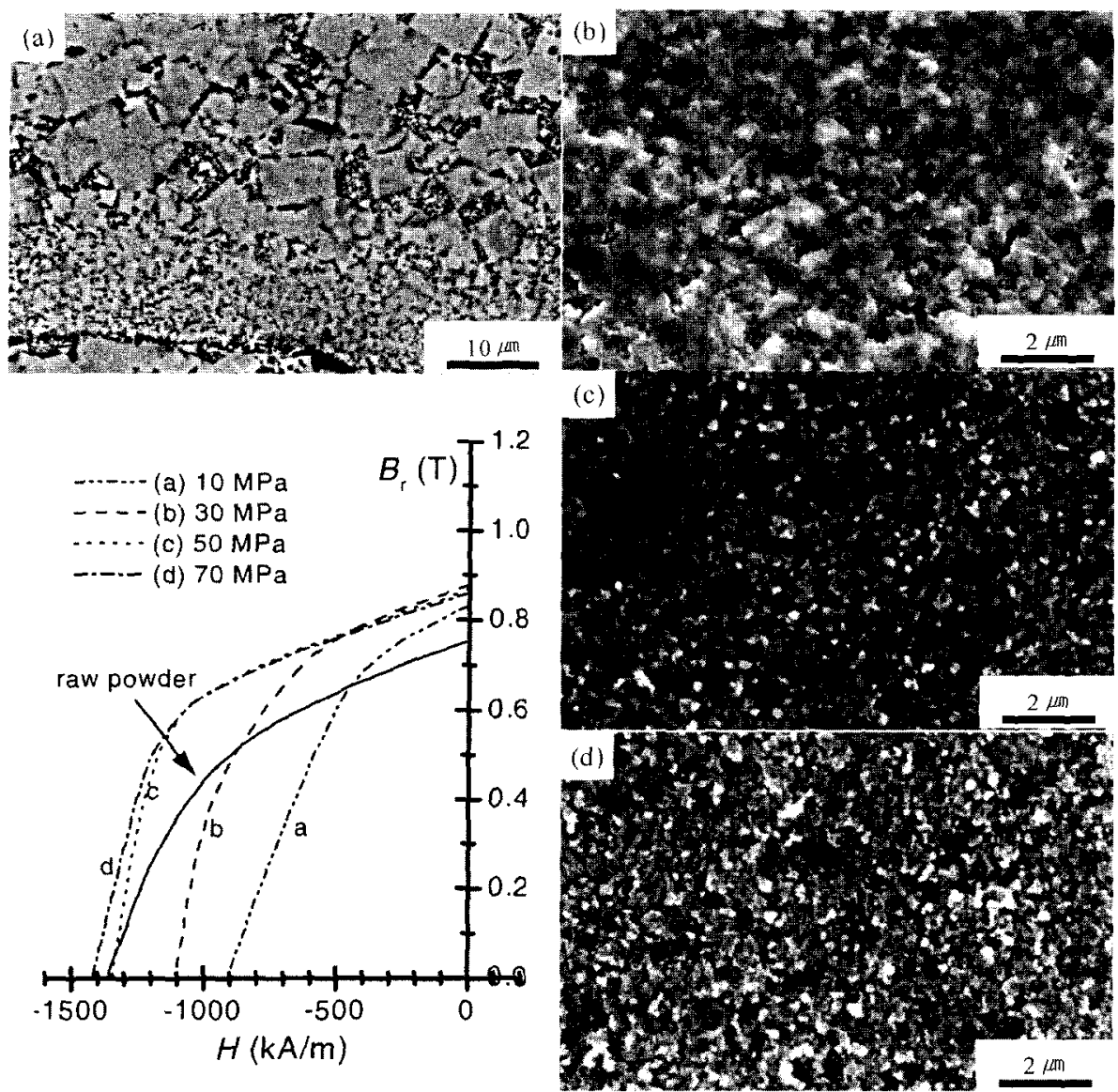

Fig. 2. Demagnetization curves and SEM images of the cross sectional surface of CA-pressed isotropic magnets obtained from MQPA powder. (a) $10 \mathrm{MPa}$, (b) $30 \mathrm{MPa}$, (c) $50 \mathrm{MPa}$ and (d) $70 \mathrm{MPa}$.

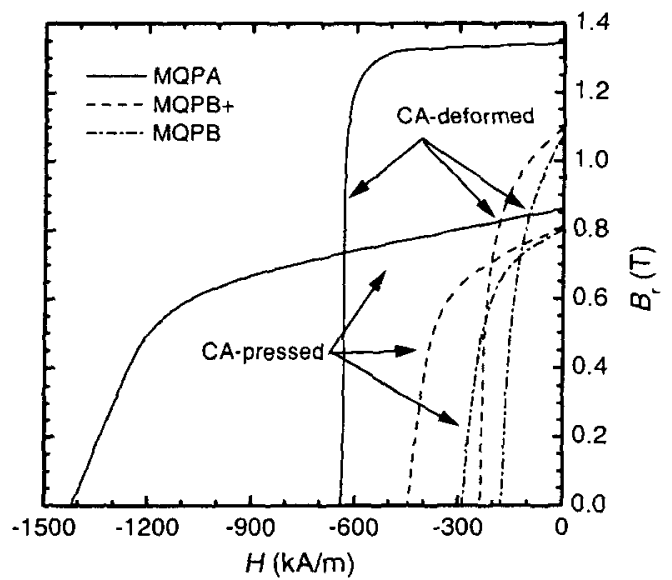

Fig. 3. Demagnetization curves measured along the press direction for the CA-pressed isotropic and CA-deformed anisotropic $\mathrm{NdFeB}$ magnets obtained from MQPA, MQPB and $\mathrm{MQPB}+$ powders.

$=45^{\circ}$ and $\alpha=80^{\circ}$, respectively. As shown in the figure, the contour for CA-deformed MQPB+ is less concentric than that for CA-deformed MQPA. It clearly illustrates the poor texture of the CA-deformed MQPB+ magnet which contains low Nd content.

Fig. 6 shows the FESEM image of the fracture surface parallel to the press direction of CA-deformed MQPA magnet. The grain structure is similar observed by Lee et al. [2] in die-upset $\mathrm{NdFeB}$ magnet. The $\mathrm{Nd}_{2} \mathrm{Fe}_{14} \mathrm{~B}$ grains appear to be platelet with $50 \sim 100 \mathrm{~nm}$ in thickness and $200 \sim 500 \mathrm{~nm}$ in length, and the c-axes of the grains are well aligned along the press direction. It indicates that the $c$-axis texture is obtained by CA-deformation as well as by the conventional hot deformation. However, the CAdeformed MQPB+ magnet is composed of two different regions, as shown in Fig. 7(b). From the magnification Fig. 7(c), the dark area (a) is revealed as the undeformed and unaligned $\mathrm{Nd}_{2} \mathrm{Fe}_{14} \mathrm{~B}$ grains. Poor workability due to the lack of Nd-rich phase probably resulted in the undeformed, so, untextured structure to which the low remanence attributes. On the other hand, the bright area (b) is identified as well-oriented $\mathrm{Nd}_{2} \mathrm{Fe}_{14} \mathrm{~B}$ platelet grains, as shown in Fig. 7(d). Surprisingly, the grain size in this area is $3 \sim 4 \mu \mathrm{m}$ in length, which is about ten times larger than that in CA-deformed MQPA magnet (Compare with Fig. 6), in spite of the original grains in both isotropic 
Table 2. The magnetic properties of the raw powders, CA-pressed and CA-deformed magnets

\begin{tabular}{|c|c|c|c|c|c|c|c|}
\hline & \multirow[t]{2}{*}{ Specimen Type } & \multicolumn{2}{|c|}{$\begin{array}{c}\text { Residual Induction } \\
B_{\mathrm{r}} \\
\end{array}$} & \multicolumn{2}{|c|}{$\begin{array}{c}\text { Intrinsic Coercivity } \\
{ }_{\mathrm{i}} H_{\mathrm{c}} \\
\end{array}$} & \multicolumn{2}{|c|}{$\begin{array}{l}\text { Energy Product } \\
\qquad(B H)_{\max }\end{array}$} \\
\hline & & (T) & $(\mathrm{kG})$ & $(\mathrm{kA} / \mathrm{m})$ & $(\mathrm{kOe})$ & $\left(\mathrm{kJ} / \mathrm{m}^{3}\right)$ & (MGOe) \\
\hline \multirow{3}{*}{ MQPA } & Raw powder & 0.76 & 7.6 & 1360 & 17.1 & 126 & 15.8 \\
\hline & CA-pressed & 0.87 & 8.7 & 1416 & 17.8 & 132 & 16.6 \\
\hline & CA-deformed & 1.34 & 13.4 & 641 & 8.1 & 341 & 42.8 \\
\hline \multirow{3}{*}{ MQPB } & Raw powder & 0.89 & 8.9 & 740 & 9.3 & 126 & 15.8 \\
\hline & CA-pressed & 0.79 & 7.9 & 287 & 3.6 & 80 & 10.1 \\
\hline & CA-deformed & 1.07 & 10.7 & 174 & 2.2 & 72 & 9.1 \\
\hline \multirow{3}{*}{ MQPB+ } & Raw powder & 0.92 & 9.2 & 760 & 9.5 & 134 & 16.8 \\
\hline & CA-pressed & 0.80 & 8.0 & 447 & 5.6 & 92 & 11.6 \\
\hline & CA-deformed & 1.09 & 10.9 & 236 & 3.0 & 111 & 13.9 \\
\hline
\end{tabular}

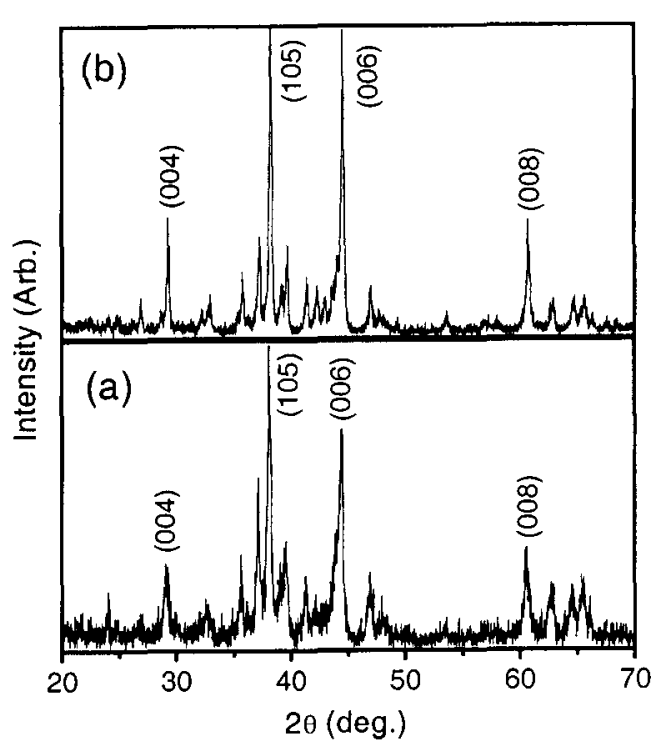

Fig. 4. XRD patterns of the surface perpendicular to the press direction for the CA-deformed magnets fabricated from MQPA (a), and MQPB+ (b) powders.

precursors were fine and uniform with $100 \sim 400 \mathrm{~nm}$ in diameter (Compare Fig. 2(d) and Fig. 7(a)).

During hot-deformation, the pre-aligned grains with $c$ axis parallel to press direction are considered to annex rapidly the inclined neighbors by solid-liquid interface diffusion through the $\mathrm{Nd}$-rich liquid phase [12]. If one accepts the pre-alignment of $\mathrm{Nd}_{2} \mathrm{Fe}_{14} \mathrm{~B}$ with $\mathrm{Nd}$-rich phase on its grain boundary is the essential condition for a nucleus, the number of nucleus in a magnet with lower $\mathrm{Nd}$ content shall be smaller than that with higher $\mathrm{Nd}$ content. A nucleus will grow until it meets the other growing nuclei, therefore, larger grains in a lower $\mathrm{Nd}$ content magnet is thought to obtain as observed in Fig. 7 (d). This large grain growth explains the considerable deterioration of the coercivity of CA-deformed MQPB and MQPB+ magnets.
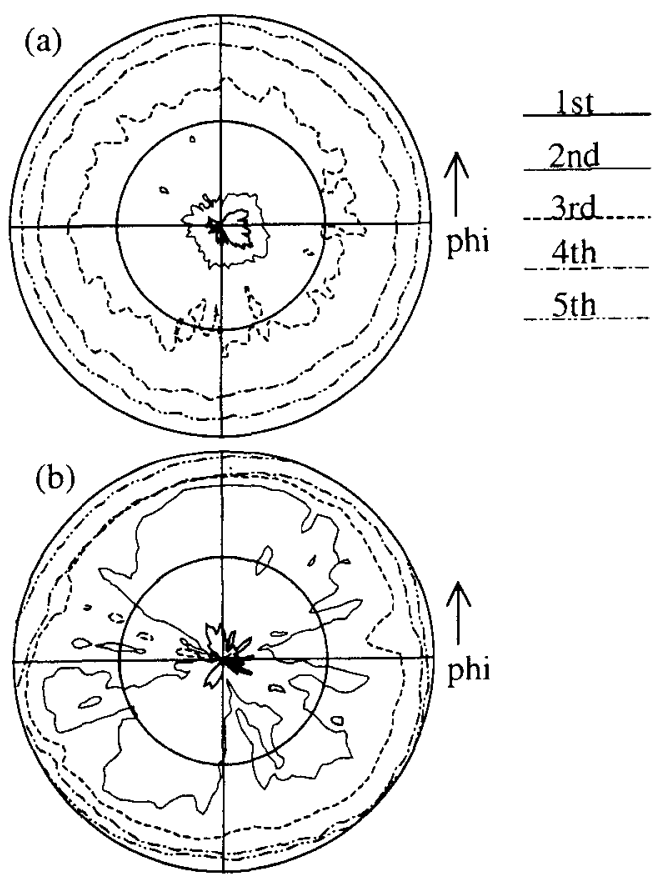

Fig. 5. The (006) pole figures of the surface perpendicular to the press direction for the CA-deformed anisotropic specimens obtained from (a) MQPA and (b) MQPB+ powders.

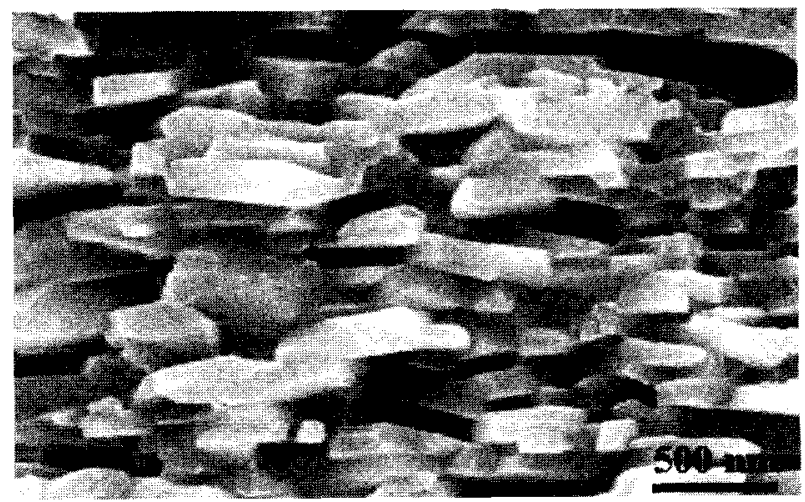

Fig. 6. The FESEM image of the fracture surface of the CAdeformed anisotropic magnet obtained from MQPA powder. 


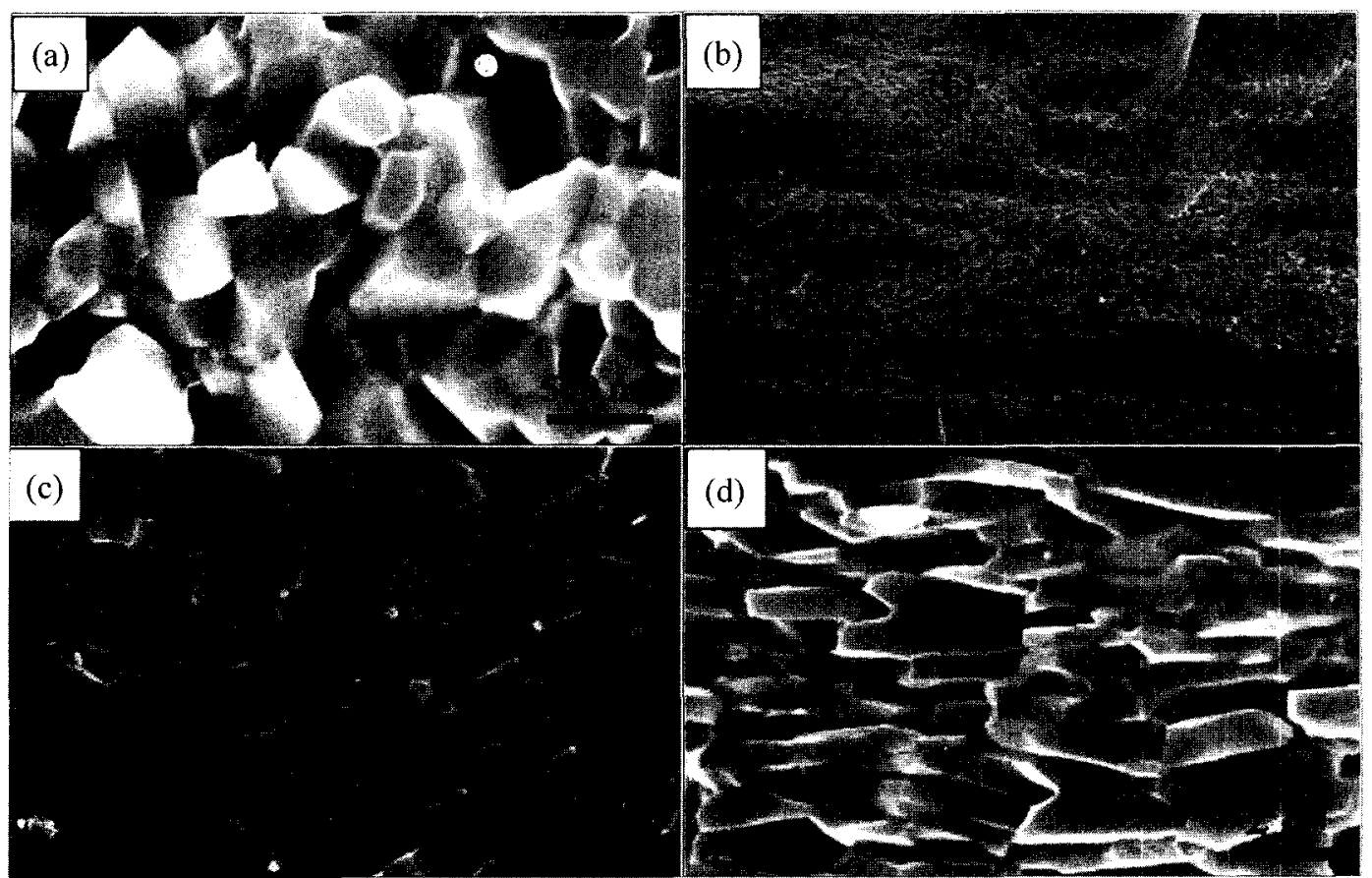

Fig. 7. FESEM images of the fracture surface parallel to the press direction for the (a) CA-pressed isotropic and (b) CA-deformed anisotropic specimens fabricated from MQPB+ powder. The two magnifications (c) and (d) correspond to the zones (a) and (b) of (b), respectively.

In conclusion, the applied pressure during CA-press is important to control the microstructure and the coercivity of CA-pressed MQPA magnets. Higher pressure results in homogeneous smaller grains and higher coercivity. The CA-deformed MQPA magnets show well-aligned fine grains, high coercivity and high remanence. However, the CA-deformed MQPB+ magnets are composed of two different regions, the area undeformed with no alignment and the other deformed, well-aligned but with large grains. The grain structure explains well the low coercivity and poor remanence of the magnets.

\section{Acknowledgement}

This work was financially supported by The Korea Institute of Science \& Technology Evaluation and Planning (KISTEP) under the International Joint R\&D Project contract No. M10011000019-01H020008800. This work was also partially supported by the Korea Science and Engineering Foundation through the Research Center for Advanced Magnetic Materials at Chungnam National University.

\section{References}

[1] R. W. Lee, Appl. Phys. Lett. 46, 790 (1985).

[2] R. W. Lee, E. G. Brewer, and N. Schaffel, IEEE Trans.
Magn. MAG-21, 1958 (1985).

[3] R. K. Mishra, T. Y. Chu, and L. K. Rabenberg, J. Magn. Magn. Mater. 84, 88 (1990).

[4] T. Saito, M. Fujita, K. Fikuoka, and Y. Syono, J. Jap. Inst. Metals 62(5), 457 (1998).

[5] S. Guruswamy, M. K. McCarter, J. E. Shield, and V. Panchnathan, J. Appl. Phys. 79(8), 4851 (1996).

[6] M. Leonowicz, W. Kaszuwara, E. Jerieroka, D. Januszewski, G. Mendora, H. A. Davies, and J. Paszula, J. Appl. Phys. 83(11), 6634 (1998).

[7] F. Fukunaga, H. Tomita, M. Wada, and F. Yamashita, J. Appl. Phys. 736(10), 6846 (1994).

[8] Z. G. Liu, M. Umemoto, S. Hirasawa, and H. Kanekyo, J. Mater. Res. 14(6), 2540 (1999).

[9] F. Ymashita, S. Hashimoto, and Y. Sasaki, IEEE Trans. Magn. 35(5), 3304 (1999).

[10] H. T. Kim, Y. B. Kim, and H. S. Kim, J. Magn. Magn. Mater. 224, 173 (2001).

[11] H. T. Kim, Y. B. Kim, and H. S. Kim, J. of Magnetics 5(4), $130(2000)$.

[12] W. C. Chang, T. B. Wu, and K. S. Liu, J. Appl. Phys. 63, 3531 (1988).

[13] R. K. Mishra, V. Panchanathan, and J. J. Croat, J. Appl. Phys. 73, 6470 (1993).

[14] W. Grünberger, D. Hinz, A. Kirchner, K. H. Müller, and L. Schultz, J. Alloys Compos. 257, 293 (1997).

[15] Lin Li and C. D. Graham, IEEE. Trans. Magn. 282, 130 (1992). 